\title{
CONSTITUINTES QUÍMICOS ISOLADOS DE SIMIRA GLAZIOVII (K. SCHUM) STEYERM. E A ATRIBUIÇÃO DOS DESLOCAMENTOS QUÍMICOS DOS ÁTOMOS DE CARBONO E HIDROGÊNIO DO ALCALÓIDE OFIORINA E SEUS DERIVADOS
}

\author{
Ana Beatriz F. D’ O. Bastos, Mário Geraldo de Carvalho* e Javier Rincón Velandia \\ Departamento de Química, Instituto de Ciências Exatas, Universidade Federal Rural do Rio de Janeiro, 23851-970 Seropédica - RJ \\ Raimundo Braz-Filho
}

Setor de Química de Produtos Naturais, LCQUI, CCT, Universidade Estadual do Norte Fluminense, 28015-620 Campos - RJ

Recebido em 18/12/00; aceito em 10/9/01

\begin{abstract}
CHEMICAL CONSTITUENTS FROM SIMIRA GLAZIOVII (K. SCHUM) STEYERM. AND ${ }^{1} \mathrm{H}$ AND ${ }^{13} \mathrm{C}$ NMR ASSIGNMENTS OF OPHIORINE AND ITS DERIVATIVES. Chromatographic fractionation of bark extracts from Simira glaziovii (Rubiaceae) afforded the steroids $\beta$-sitostenone, stigmastenone, $\beta$-sitosterol and stigmasterol, methyl trans-4-hidroxy-3-methoxycinamate (1), the alkaloids harmane (2) and the new stereoisomer of ophiorine B (3). The structures were established by ${ }^{1} \mathrm{H}$ and ${ }^{13} \mathrm{C}$ NMR, including 2D techniques and mass spectral analysis, of the natural products and pentaacetyllyalosidic acid (4a) and $\beta$-carboline monoterpene tetraacetylglucoside $(\mathbf{5}, 1,22$-lactamlyaloside) derivatives obtained by chemical transformations.
\end{abstract}

Keywords: Simira glaziovii; Rubiaceae; steroids; harmane; (-)-ophiorine.

\section{INTRODUÇÃO}

Plantas do gênero Simira vem despertando interesse devido principalmente às atividades fototóxicas apresentadas por alguns de seus constituintes químicos e pelas informações etnomédicas sobre o tratamento de manchas na cavidade oral e dentária ${ }^{1}$ com cascas frescas de $S$. rubescens. A literatura registra resultados de estudos químicos das espécies $S$. Maxonii $i^{2-4}, S$. mexicana ${ }^{5}$, S. Salvadorensis ${ }^{6}$ e $S$. rubra $^{6}$. Foram identificados, além de policetídeos, alcalóides fotossenbilizantes furoquinolínicos e $\beta$-carbolínicos ${ }^{5,6}$. Os alcalóides harmana, maxonina e estrictosamida aparecem como os mais comuns neste gênero ${ }^{7}$.

Este artigo descreve o primeiro estudo fitoquímico de um espécimen de $S$. glaziovii (K. Schum) Steyerm, que permitiu o isolamento e a caracterização dos esteróides $\beta$-sitostenone, estigmastenone, $\beta$-sitosterol and estigmasterol, 3,4-dimetoxicinamato de metila (1), o alcalóide harmana (2), conhecido como 1-metil- $\beta$-carbolina, aribina, loturina ou passiflorina, isolado anteriormente de Arariba rubra e Passiflora incarnata ${ }^{7}$, e o alcalóide ofiorina B (3), encontrado em Ophiorrhiza japonica e $O$. kuroiwai (Rubiaceae) ${ }^{8}$. Com exceção do alcalóide harmana, os demais constituintes estão sendo caracterizados pela primeira vez no gênero Simira.

$\mathrm{O}$ tratamento do alcalóide ofiorina (3), epímero no átomo de carbono C-16 da ofiorina A (3a), com diazometano $\left(\mathrm{CH}_{2} \mathrm{~N}_{2}\right)$ forneceu o produto rearranjado conhecido como lialosídeo (4). A preparação dos dois novos derivados da ofiorina $\mathrm{B}(\mathbf{4 a}$ e 5) e a análise dos dados espectrométricos de $\mathrm{RMN}{ }^{1} \mathrm{H}$ e ${ }^{13} \mathrm{C}(1 \mathrm{D}$ e $2 \mathrm{D})$ permitiu caracterizar o alcalóide $\mathbf{3}$ e estabelecer a completa atribuição dos deslocamentos químicos dos átomos de hidrogênio e carbono, eliminandose inclusive equívocos registrados na literatura.

\section{RESULTADOS E DISCUSSÃO}

O fracionamento cromatográfico do extrato metanólico das cascas de S. glaziovii forneceu além dos alcalóides harmana (2) e (-)-

\footnotetext{
*e-mail: mgeraldo@ufrrj.br
}<smiles>COC(=O)/C=C/c1ccc(OC)c(OC)c1</smiles>

1<smiles>Cc1nccc2c1[nH]c1ccccc12</smiles>
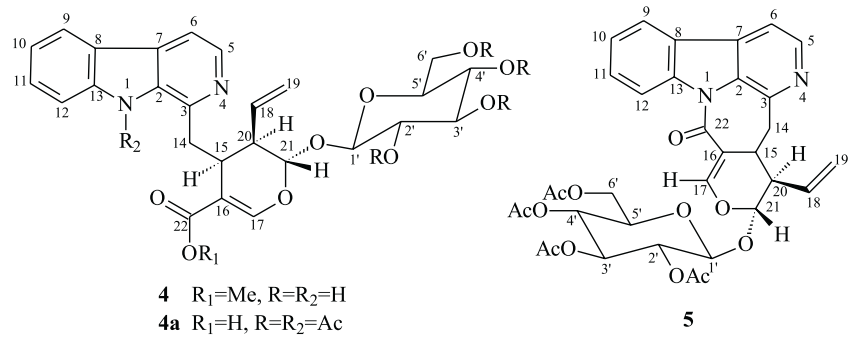

ofiorina (3), a mistura de $\beta$-sitosterol, estigmasterol e 3,4-dimetoxicinamato de metila (1). O alcalóde $\mathbf{3}$ apresentou dados espectrométricos semelhantes aos da (+)-ofiorina $\mathrm{B}$ já registrada na literatu$\mathrm{ra}^{8}$ e, levando em consideração de que se trata de um glicosideo natural, identificou-o como o novo estereoisômero deste alcalóide. Do extrato hexânico foi identificada a mistura de sitostenona e estigmastenona. Os derivados $\mathbf{4 a}$ e $\mathbf{5}$ estão sendo registrados pela primeira vez na literatura.

Os esteróides, o éster 1 e o alcalóide harmana (2) foram identificados através da análise dos dados fornecidos pelos espectros IV e $\mathrm{RMN}{ }^{1} \mathrm{H}$ e ${ }^{13} \mathrm{C}$ comparados com valores registrados na literatura ${ }^{9-15}$.

$\mathrm{O}$ espectro de $\mathrm{RMN}{ }^{1} \mathrm{H}$ de $\mathbf{3}$ mostrou sinais de seis hidrogênios aromáticos da unidade $\beta$-carbolínica. Picos transversais correspondentes às interações heteronucleares de átomos de carbono e hidrogênio observados nos espectros 2D HMQC $\left({ }^{1} \mathrm{~J}_{\mathrm{CH}}\right)$ e $\left.\operatorname{HMBC}\left({ }^{2,3} \mathrm{~J}_{\mathrm{CH}}\right)\right]$ permiti- 
ram identificar os sinais dos carbonos hidrogenados e quaternários desta unidade aromática $\left(\mathrm{C}_{11} \mathrm{H}_{6} \mathrm{~N}_{2}\right)$, Tabela 1 . Além disso, a análise dos espectros de $\mathrm{RMN}{ }^{1} \mathrm{H} \mathrm{e}{ }^{13} \mathrm{C}(1 \mathrm{D}$ e $2 \mathrm{D})$ conduziu às seguintes deduções: a) A presença do grupo vinila foi reconhecida pelos sinais dos carbonos: metínico $\mathrm{CH}-18$ [ $\delta_{\mathrm{C}} 134,70$ e $\delta_{\mathrm{H}} 5,76(\mathrm{ddd}, 6,4,10,8$ e 17,2 Hz)] e metilênico $\mathrm{CH}_{2}-19\left[\delta_{\mathrm{C}} 120,90\right.$ e $\delta_{\mathrm{H}} 5,25(\mathrm{~d}, 10,8 \mathrm{~Hz})$ e $5,27(\mathrm{~d}, 17,2$ $\mathrm{Hz}$ )] b) A unidade de $\beta$-glicopiranosila foi proposta através dos sinais dos átomos de carbono oxigenados metínicos $\mathrm{CH}-1$ ' $\left[\delta_{\mathrm{C}} 99,74\right.$ e $\delta_{\mathrm{H}} 4,34$ (d, 8,8 Hz, H-1' em posição axial), carbono anomérico], $\mathrm{CH}-2$ ' $\left[\delta_{\mathrm{C}} 73,15\right.$ e 2,90 (t, J=8,8 Hz)], CH-3' [ $\delta_{\mathrm{C}} 76,76$ e $\left.\delta_{\mathrm{H}} 3,10(\mathrm{~m})\right], \mathrm{CH}-4$ ' $\left[\delta_{\mathrm{C}} 69,80 \mathrm{e}\right.$ $\left.\delta_{\mathrm{H}} 3,00(\mathrm{~m})\right]$ e CH-5' $\left[\delta_{\mathrm{C}} 77,00\right.$ e $\left.\delta_{\mathrm{H}} 2,80(\mathrm{~m})\right]$ e metilênico $\mathrm{CH}_{2}-6^{\prime}\left[\delta_{\mathrm{C}}\right.$ 60.57 e $\delta_{\mathrm{H}} 3,51(\mathrm{~m})$ e $\left.3,20(\mathrm{~m})\right]$. A comparação destes dados espectrométricos com valores registrados na literatura ${ }^{8}$ para alcalóides $\beta$ carbolínicos glicosilados contendo grupo vinila permitiu destacar a presença de sinais remanescentes nos espectros de RMN ${ }^{13} \mathrm{C}$ em $\delta_{\mathrm{CH}} 94,22$, 89,00 (carbinólicos), 47,64, 46,59 e 31,04 e $\delta_{\mathrm{C}}$ 175,60 (compatível para ácido carboxílico). Estes dados revelaram-se semelhantes aos deslocamentos químicos descritos para os alcalóides ofiorinas A e B (Tabela 1). Surpreendentemente, o sinal do $\mathrm{CH}_{2}-14$ não foi detectado no espectro registrado em $\mathrm{D}_{2} \mathrm{O}$, sendo observado em $\delta_{\mathrm{C}} 24,00\left(\mathrm{CH}_{2}\right)$ no espectro registrado em MeOH- $d_{4}$. O pico em $m / z 513\left([\mathrm{M}+1\}, 55 \%, \mathrm{C}_{26} \mathrm{H}_{29} \mathrm{O}_{9} \mathrm{~N}_{2}\right.$, Esquema 1) observado no espectro de massas (FAB-MS) revelou-se em acordo com a formula molecular $\mathrm{C}_{26} \mathrm{H}_{28} \mathrm{O}_{9} \mathrm{~N}_{2}$ correspondente às estrutu- ras destas substâncias alcaloídicas. A análise comparativa dos dados obtidos dos espectros de $\mathrm{RMN}^{1} \mathrm{H}^{13} \mathrm{C}$ registrados em $\mathrm{D}_{2} \mathrm{O}$ (Tabela 1), incluindo-se experimentos 1D e 2D (HMQC e HMBC), permitiu distinguir entre os dois epímeros ofiorinas B (3) e A (3a), caracterizando-se o alcalóide indólico isolado de Simira glaziovii como ofiorina B (3) e fazer as atribuções inequívocas dos deslocamentos químicos dos carbonos desta substância. A leitura da rotação óptica de 3, $[\alpha]_{\mathrm{D}}:-13,95$ (c $0,043, \mathrm{MeOH})$, a igualdade entre os valores dos deslocamentos químicos de ${ }^{1} \mathrm{H} \mathrm{e}{ }^{13} \mathrm{C}$ com os da ofiorina $\mathrm{B}$ registrada na literatura ${ }^{8}$ e levando em consideração de se tratar de um glicosídeo natural postulou-se a configuração (-)-ofiorina para a substância isolada de $S$. glaziovii e correspondendo a um novo estereoisômero da (+)ofiorina B registrada na literatura com $[\alpha]_{D}:+18,0^{8}$.

A análise detalhada dos espectros de massas confirmou a facilidade de fragmentação do alcalóide $\mathbf{3}$ no espectrômetro de massas. $\mathrm{O}$ espectro obtido com ionização por FAB-MS (glicerol como matriz, Esquema 1) revelou diferenças significativas quando comparado com o registrado por ionização química (CI-MS, Esquema 2). O FAB-MS mostrou o pico $[\mathrm{M}+1] \mathrm{em} m / z 513\left(55 \%, \mathrm{C}_{26} \mathrm{H}_{29} \mathrm{O}_{9} \mathrm{~N}_{2}\right)$, e picos adicionais em $\mathrm{m} / \mathrm{z} 469\left[5 \%, \mathrm{~m} / \mathrm{z} 513-44\left(\mathrm{CO}_{2}\right), \mathrm{C}_{25} \mathrm{H}_{29} \mathrm{O}_{7} \mathrm{~N}_{2}\right], 351[15 \%, \mathrm{~m} /$ $\left.z 513-162\left(\mathrm{C}_{6} \mathrm{H}_{10} \mathrm{O}_{5}\right), \mathrm{C}_{20} \mathrm{H}_{19} \mathrm{O}_{4} \mathrm{~N}_{2}\right], 307\left[27 \%, \mathrm{~m} / z, 513-44\left(\mathrm{CO}_{2}\right)-\right.$ $162\left(\mathrm{C}_{6} \mathrm{H}_{10} \mathrm{O}_{5}\right)$ e/ou $\left.m / z 513-162\left(\mathrm{C}_{6} \mathrm{H}_{10} \mathrm{O}_{5}\right)-44\left(\mathrm{CO}_{2}\right), \mathrm{C}_{19} \mathrm{H}_{19} \mathrm{O}_{2} \mathrm{~N}_{2}\right]$, $219\left(59 \%, \mathrm{C}_{15} \mathrm{H}_{11} \mathrm{~N}_{2}\right), 182\left(60 \%, \mathrm{C}_{12} \mathrm{H}_{10} \mathrm{~N}_{2}\right)$ e $133\left[100 \%, \mathrm{C}_{5} \mathrm{H}_{9} \mathrm{O}_{4}\right]$,

Tabela 1. Dados de $\mathrm{RMN}^{1} \mathrm{H}(400 \mathrm{MHz})$ e ${ }^{13} \mathrm{C}(100 \mathrm{MHz})$ de 3 e comparação com os deslocamentos químicos de carbono-13 das ofiorinas A (3a) e $\mathrm{B}(3)$ registrados na literatura ${ }^{8}\left(\text { solvente } \mathrm{D}_{2} \mathrm{O}\right)^{*}$

\begin{tabular}{|c|c|c|c|c|c|c|}
\hline \multirow[b]{3}{*}{$\mathrm{C}$} & \multirow{2}{*}{\multicolumn{2}{|c|}{$\delta_{\mathrm{C}}{ }^{8}$}} & \multicolumn{4}{|c|}{3} \\
\hline & & & \multicolumn{2}{|r|}{ HMQC } & \multicolumn{2}{|c|}{ HMBC } \\
\hline & $\mathbf{3 a}$ & 3 & $\delta_{\mathrm{C}}$ & $\delta_{\mathrm{H}}$ & ${ }^{2} \mathbf{J}_{\mathrm{CH}}$ & ${ }^{3} \mathbf{J}_{\mathrm{CH}}$ \\
\hline 2 & 134,8 & 135,6 & 135,9 & - & - & H-6 \\
\hline 3 & 146,6 & 146,4 & 138,8 & - & - & $\mathrm{H}-5, \mathrm{H} 17$ \\
\hline 5 & 136,9 & 136,8 & 133,3 & $8,6(\mathrm{~d}, \mathrm{~J}=6,8)$ & H-6 & H-17 \\
\hline 6 & 135,9 & 135,8 & 118,0 & $8,3(\mathrm{~d}, \mathrm{~J}=6,8)$ & $\mathrm{H}-5$ & - \\
\hline 7 & 140,2 & 140,4 & 130,2 & - & - & H-5,H9 \\
\hline 8 & 122,1 & 121,9 & 119,2 & - & - & H-6,H-10,H-12 \\
\hline 9 & 124,6 & 124,5 & 123,4 & $8,1(\mathrm{~d}, \mathrm{~J}=8,0)$ & - & H-11 \\
\hline 10 & 119,2 & 119,2 & 122,7 & $7,1(\mathrm{t}, \mathrm{J}=8,0)$ & - & \\
\hline 11 & 125,5 & 125,5 & 132,6 & $7,4(\mathrm{t}, \mathrm{J}=8,0)$ & - & - \\
\hline 12 & 115,4 & 115,3 & 113,3 & $7,6(\mathrm{~d}, \mathrm{~J}=8,0)$ & $\mathrm{H}-11$ & H-10 \\
\hline 13 & 135,8 & 134,7 & 144,1 & - & - & H-9,H-11 \\
\hline 14 & 26,0 & 29,1 & $24,0^{\mathrm{b}}$ & 3,5 & - & - \\
\hline 15 & 33,0 & 33,3 & 31,0 & $3,0(\mathrm{~m})$ & - & \\
\hline 16 & 49,8 & 47,4 & 46,6 & 3,3 & - & $\mathrm{H}-14$ \\
\hline 17 & 91,7 & 91,5 & 89,0 & $6,6(\mathrm{sl})$ & $\mathrm{H}-16$ & $\mathrm{H}-5$ \\
\hline 18 & 134,8 & 134,8 & 134,7 & $5,7(\mathrm{dd}, \mathrm{J}=6,4 ; 10,8 ; 17,2)$ & $2 \mathrm{H}-18$ & - \\
\hline \multirow[t]{2}{*}{19} & 121,9 & 122,3 & 120,9 & $5,2(\mathrm{dl}, \mathrm{J}=10,8)$ & & \\
\hline & & & & $5,3(\mathrm{dl}, \mathrm{J}=17,2)$ & & \\
\hline 20 & 48,8 & 46,0 & 47,6 & $2,8(\mathrm{~m})$ & - & 2H-19,H-16,H-1, \\
\hline 21 & 98,2 & 98,2 & 94,2 & $4,6(\mathrm{~d}, \mathrm{~J}=9,6)$ & - & H-17,H-19 \\
\hline 22 & 176,9 & 177,4 & 175,6 & - & H-16 & - \\
\hline 1 ' & 101,8 & 102,2 & 99,7 & $4,4(\mathrm{~d}, \mathrm{~J}=8,8)$ & $\mathrm{H}-2$ & - \\
\hline 2 ' & 75,4 & 75,4 & 73,5 & $2,9(\mathrm{t}, \mathrm{J}=8,8)$ & H-3' & - \\
\hline $3^{\prime}$ & 78,8 & 78,9 & 76,6 & $3,1(\mathrm{~m})$ & $\mathrm{H}-2$ & - \\
\hline $4^{\prime}$ & 72,2 & 72,2 & 69,8 & $3,0(\mathrm{~m})$ & H-3' & \\
\hline 5 & 78,4 & 78,3 & 77,0 & $2,8(\mathrm{~m})$ & $\mathrm{H}-4$ & \\
\hline \multirow[t]{2}{*}{$6^{\prime}$} & 63,3 & 63,2 & 60,6 & $3,5(\mathrm{~m})$ & - & - \\
\hline & & & & $3,2(\mathrm{~m})$ & - & - \\
\hline
\end{tabular}

*Os sinais de átomos de carbono $\mathrm{C}, \mathrm{CH}$ e $\mathrm{CH}_{3}$ foram reconhecidos pela análise comparativa dos espectros de $\mathrm{RMN}{ }^{13} \mathrm{C}-\mathrm{HBBD}$ e $\mathrm{RMN}{ }^{13} \mathrm{C}$ DEPT. O espectro $2 \mathrm{D}^{1} \mathrm{H}-{ }^{1} \mathrm{H}$-COSY foi também utilizado. Os deslocamentos químicos e constantes de acoplamento (J) dos sinais de hidrogênio foram deduzidos do espectro $1 \mathrm{D}$ de $\mathrm{RMN}^{1} \mathrm{H}$. ${ }^{\mathrm{b}}$.sinal observado no espectro obtido em MeO- $\mathrm{D}_{4}$. 


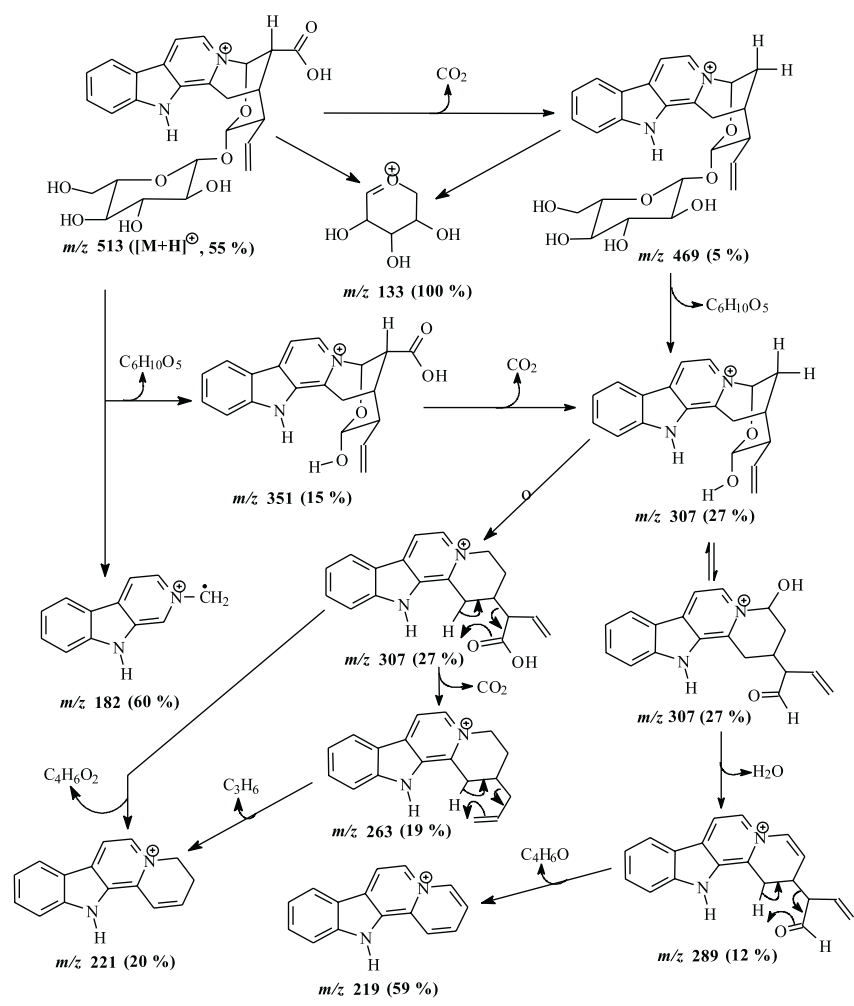

Esquema 1. Proposta de fragmentação do alcalóide 3 no espectrômetro de massas usando ionização por FAB (modo positivo, glicerol como matriz)

como resumido no Esquema 1. No CI-MS o pico $m / z, 513([\mathrm{M}+1])$ não foi observado e os picos principais apareceram em $\mathrm{m} / z, 335$ [10\%, $m / z 513\left(\mathrm{C}_{26} \mathrm{H}_{29} \mathrm{O}_{9} \mathrm{~N}_{2}\right)-178\left(\mathrm{C}_{6} \mathrm{H}_{10} \mathrm{O}_{6}\right), 329[27 \%, \mathrm{~m} / \mathrm{z}, 513-184$ $\left.\left(\mathrm{C}_{5} \mathrm{H}_{12} \mathrm{O}_{7}\right), \mathrm{C}_{21} \mathrm{H}_{17} \mathrm{O}_{2} \mathrm{~N}_{2}\right], 317\left[92 \%, \mathrm{~m} / \mathrm{z} 335-18\left(\mathrm{H}_{2} \mathrm{O}\right), \mathrm{C}_{20} \mathrm{H}_{17} \mathrm{O}_{2} \mathrm{~N}_{2}\right]$ e $307\left[100 \%, \mathrm{~m} / \mathrm{z} 335-28\left(\mathrm{CH}_{2}=\mathrm{CH}_{2}\right), \mathrm{C}_{18} \mathrm{H}_{15} \mathrm{O}_{3} \mathrm{~N}_{2}\right.$ ] (Esquema 2). Todos estes picos podem ser atribuídos a fragmentos formados através de reações relativamente simples, informativas e previstas pela estrutura 3, como revelam resumidamente os Esquemas 1 e 2 .

A reação de 3 com diazometano forneceu o produto rearranjado lialosilato de metila $(4)^{16}$, como descrito na literatura ${ }^{8}$. A formação deste produto serviu como um dado adicional para confirmação da estrutura proposta.

A acetilação de $\mathbf{3}$ com anidrido acético na presença de piridina forneceu dois produtos (4a e 5). A análise dos dados espectrométricos obtidos por RMN ${ }^{1} \mathrm{H}$ e ${ }^{13} \mathrm{C}$ e comparação com os valores atribuídos para o alcalóide $\mathbf{3}$ permitiu propor as estruturas $\mathbf{4 a}$ e $\mathbf{5}$ para os derivados acetilados (Tabelas 2 e 3 ).

As informações obtidas dos espectros 2D (HMQC e HMBC) dos produtos 4a e 5 permitiram identificar os sinais dos átomos de carbono hidrogenados e quaternários da unidade $\beta$-carbolínica e verificar as diferenças correspondentes às estruturas de $\mathbf{3}$ e $\mathbf{4 a}$ e $\mathbf{5}$ (Tabelas 2 e 3). As principais diferenças entre os dados dos produtos $\mathbf{4 a}$ e 5 e a ofierina B (3) podem ser resumidas com base na: a) ausência dos sinais de átomos de carbono em $\delta_{\mathrm{C}} 89,0(\mathrm{CH}-17)$ e 46,59 $(\mathrm{CH}-$ 16) e hidrogênio em $\delta_{\mathrm{H}} 6,6$ (sl, H-17) e 3,3 (m, H-16) nos espectros de 4a e 5; b) presença dos sinais do $\mathrm{CH}-17$ [4a/5: $\delta_{\mathrm{C}} 147,6 / 150,9$ e $\left.\delta_{\mathrm{H}} 6,95(\mathrm{~d}, 2,8 \mathrm{~Hz}) / 7,80(\mathrm{~d}, 2,8 \mathrm{~Hz})\right]$ e $\mathrm{C}-16\left(\mathbf{4 a} / \mathbf{5}: \delta_{\mathrm{C}} 114,6 / 112,6\right)$; c) ausência do sinal em $\delta_{\mathrm{C}} 175,6(\mathrm{C}-22)$ nos espectros dos produtos (4a e 5) e a presença dos sinais de átomos de carbono carbonílicos (C-22) envolvidos em sistemas conjugados $\alpha, \beta$-insaturados (4a/5: $\delta_{\mathrm{C}}$ 167,7/166,3). Os espectros HMBC revelaram picos transversais correlacionando interação spin-spin através de três ligações $\left({ }^{3} \mathbf{J}_{\mathrm{CH}}\right)$ entre os sinais de C-22 (4a/5: $\left.\delta_{\mathrm{C}} 167,7 / 166,3\right)$ e $\mathrm{H}-17$ [4a/5: $\delta_{\mathrm{H}} 6,95$

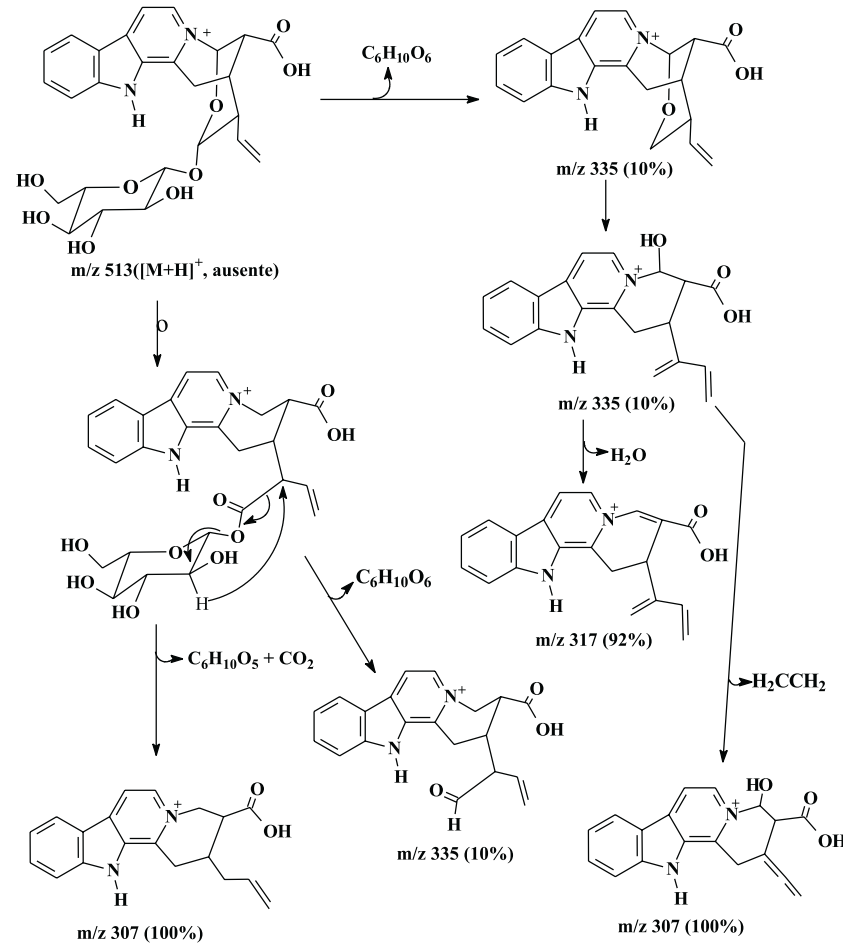

Esquema 2. Proposta de fragmentação do alcalóide 3 no espectrômetro de massas usando ionização química (CI)

(d, 2,8 Hz)/7,80 (d, 2,8Hz)] (item b); d) os deslocamentos químicos e a multiplicidade dos sinais dos carbonos metínicos e metilênicos da unidade $\beta$-glicopiranosídica revelaram-se compatíveis com a presença dos quatro grupos acetoxílicos (Tabelas 2 e 3); e) a análise adicional da região de absorção dos grupos acetoxílicos $\left[(\mathrm{AcO})_{5} \mathrm{em}\right.$ 4a e $(\mathrm{AcO})_{4}$ em 5] nos espectros HMQC e HMBC, facilitada através de expansões espectrais, permitiu reconhecer os sinais dos átomos de carbono e hidrogênio ligados entre si $\left({ }^{1} \mathrm{~J}_{\mathrm{CH}}\right)$ nos grupos metílicos e dos átomos de carbono carbonílicos com os hidrogênios metílicos $\left({ }^{2} \mathrm{~J}_{\mathrm{CH}}\right)$ das unidades acetoxílicas.

Todos estes dados e a avaliação da reação realizada por tratamento de $\mathbf{3}$ em meio básico permitiram postular a formação de $\mathbf{4}$ como consequência da abstração inicial do H-16, a formação ligação dupla entre os átomos de carbono C-16 e C-17 e a neutralização da carga positiva localizada no nitrogênio N-4. A acetilação posterior de $\mathbf{4 a}$ produziu o derivado $\mathbf{5}$. O ataque nucleofílico do átomo de nitrogênio N-1 no carbono carbonílico C-22 justifica a ciclização e, consequentemente, a formação do produto 5 (Tabelas 2 e 3).

\section{PARTE EXPERIMENTAL}

Procedimentos experimentais gerais: Os pontos de fusão foram determinados em placa aquecida de Kofler e não foram corrigidos. Os espectros infra-vermelho (IV) foram registrados em pastilhas de $\mathrm{KBr}$ no espectrômetro 1420 da Parkin-Elmer. Os espectros de RMN foram registrados nos aparelhos UN-400 $\left({ }^{1} \mathrm{H}: 400 \mathrm{MHz}\right.$ e ${ }^{13} \mathrm{C}: 100$ $\mathrm{MHz})$ da Varian e AC-200 ( ${ }^{1} \mathrm{H}: 200,13 \mathrm{MHz}$ e $\left.{ }^{13} \mathrm{C}: 50,3 \mathrm{MHz}\right) \mathrm{da}$ Bruker. Os espectros de massas foram obtidos em um espectrômetro VG-Quatro. Nas separações cromatográficas em coluna e camada fina analítica e preparativa usou-se sílica gel da Aldrich ou Merck com granulação adequada. As placas cromatográficas foram reveladas com luz UV ( $\left.\lambda_{\max } 254 \mathrm{~nm}\right)$, vapores de iodo e/ou solução alcoólica de vanilina e ácido sulfúrico. 
Tabela 2. Dados de $\mathrm{RMN}{ }^{1} \mathrm{H}(400 \mathrm{MHz})$ e ${ }^{13} \mathrm{C}(100 \mathrm{MHz})$ do produto acetilado $4 \mathbf{a}^{*}$

\begin{tabular}{|c|c|c|c|c|}
\hline \multirow[b]{2}{*}{$\mathrm{C}$} & \multicolumn{2}{|c|}{ HMQC } & \multirow{2}{*}{$\begin{array}{c}\text { HMBC } \\
{ }^{2} \mathrm{~J}_{\mathrm{CH}} \mathrm{e}^{3} \mathrm{~J}_{\mathrm{CH}}\end{array}$} & \multirow[b]{2}{*}{${ }^{1} \mathrm{Hx}{ }^{1} \mathrm{H}-\mathrm{COSY}$} \\
\hline & $\delta_{\mathrm{C}}$ & $\delta_{\mathrm{H}}$ & & \\
\hline 2 & 134,7 & - & H-6 & \\
\hline 3 & 144,3 & - & $\mathrm{H}-5$ & \\
\hline 5 & 143,1 & $8,56(\mathrm{~d} ; 4,8)$ & & H-6 \\
\hline 6 & 113,2 & $7,45(\mathrm{~d} ; 4,8)$ & H-5 & $\mathrm{H}-5$ \\
\hline 7 & 133,4 & - & H-9; H-5 & \\
\hline 8 & 124,2 & - & H-10 & \\
\hline 9 & 120,8 & $7,99(\mathrm{~d} ; 8,0)$ & $\mathrm{H}-11$ & H-10; \\
\hline 10 & 1246 & $7,44(\mathrm{t} ; 8,0)$ & $\mathrm{H}-12$ & H-9; H-11 \\
\hline 11 & 130,2 & $7,61(\mathrm{t} ; 8,0)$ & H-9 & $\mathrm{H}-10 ; \mathrm{H}-12$ \\
\hline 12 & 118,5 & $8,58(\mathrm{~d} ; 8,0)$ & H-10 & H-11 \\
\hline 13 & 140,7 & - & H-11; H-9 & \\
\hline 14 & 33,9 & $2,20(\mathrm{~m})$ & & \\
\hline 15 & 34,8 & $4,50$ (ddd; 4,$4 ; 3,2 ;-)$ & & $\mathrm{H}-20$ \\
\hline 16 & 114,6 & - & $\mathrm{H}-17 ; \mathrm{H}_{2} 0$ & \\
\hline 17 & 1476 & $6,95(\mathrm{~d} ; 2,8)$ & $\mathrm{H}-21$ & \\
\hline 18 & 131,5 & $\begin{array}{c}5,42 \text { (ddd; } 16,0 \\
10,0 ; 8,0)\end{array}$ & $\mathrm{H}-21$ & H-19; H-20 \\
\hline 19 & 120,4 & $\begin{array}{c}5,30(\mathrm{dd} ; 16,0 ; 2,0) \\
5.40(\mathrm{~m})\end{array}$ & & $\mathrm{H}-19 \mathrm{~b}, 19 \mathrm{a}$ \\
\hline 20 & 42,9 & $5,04(\mathrm{dd} ; 8,0 ; 2,0)$ & & \\
\hline 21 & 95,5 & $2,97(\mathrm{dm} ; 6,0)$ & & H-15; H-18 \\
\hline 22 & 167,7 & - & $\mathrm{H}-17$ & \\
\hline 1 ' & 95,8 & $4,96(\mathrm{~d} ; 8,0)$ & & H-2' \\
\hline $2^{\prime}$ & 70,3 & $5,04(\mathrm{t} ; 8,0)$ & & H-1'; H-3' \\
\hline $3^{\prime}$ & 72,2 & $5,30(\mathrm{t} ; 9,6)$ & & H-2'; H-4' \\
\hline 4 ' & 68,2 & $5,13(\mathrm{t} ; 9,0)$ & & $\mathrm{H}-3$ '; H-5, \\
\hline 5 & 72,3 & $3,80(\mathrm{dm} ; 12,8 ; 2,4)$ & & H-4'; H-6' \\
\hline $6^{\prime}$ & 61,7 & $4.20(\mathrm{dd} ; 12,8 ; 2,4)$ & & H-5'; H-6’b \\
\hline & & $4,37(\mathrm{dd}, 12,8 ; 4,8)$ & & H-5'; H-6'a \\
\hline $\mathrm{H}_{3} \mathrm{CCO}$ & 20,$9 ; 20,7 ; 20,6 ; 20,5 ; 17,8$ & 2,$36 ; 2,23 ; 2,10 ; 2,08 ; 1,84$ & & \\
\hline $\mathrm{H}_{3}^{3} \mathrm{C} \underline{\mathrm{CO}}$ & 170,$6 ; 170,0 ; 169,7 ; 169,5 ; 169,3$ & & $\underline{\mathrm{H}}_{3} \mathrm{C}-\mathrm{CO}$ & \\
\hline
\end{tabular}

*Os sinais de átomos de carbono $\mathrm{C}, \mathrm{CH}$ e $\mathrm{CH}_{3}$ foram reconhecidos pela análise comparativa dos espectros de $\mathrm{RMN}{ }^{13} \mathrm{C}-\mathrm{HBBD}$ e $\mathrm{RMN}{ }^{13} \mathrm{C}$ DEPT. Os deslocamentos químicos e constantes de acoplamento ( $\mathrm{J}$ em Hz entre parenteses) dos sinais de hidrogênio foram deduzidos do espectro $1 \mathrm{D}$ de $\mathrm{RMN}{ }^{1} \mathrm{H}$.

Planta: O material vegetal foi coletado na reserva florestal de Tinguá, Nova Iguaçu, RJ, Brasil pelo Professor S. J. da Silva Neto (IB - UFRRJ). A identificação foi feita através de comparação com a excicata ( $\mathrm{N}^{\circ}$ 1513) depositada no herbário do Departamento de Botânica do Instituto de Biologia - UFRRJ.

Extração e separação: As cascas de S. glaziovii (K. Schum) Steyerm. foram secas e moídas $(930 \mathrm{~g})$ e submetidas à extração através de maceração com hexano até exaustão e depois com metanol. As soluções dos extratos foram concentradas em evaporador rotativo sob vácuo, obtendo-se os resíduos dos extratos hexânico (SGCH, $600 \mathrm{mg}$ ) e metanólico (SGCM, 95,7g).

O extrato metanólico foi fracionado por cromatografia em coluna de sílica gel. Foram coletadas 71 frações e reunidas em grupos após análise através de cromatografia em camada fina analítica. Os grupos de frações foram monitorados por bioensaio de letalidade com Artemia salina ${ }^{17}$, identificando-se as propriedades tóxicas das frações investigadas. O grupo das frações reunidas 26-32 revelou maior toxicidade $\left(\mathrm{DL}_{50}=223 \mathrm{ppm}\right)$, enquanto os demais não apresentaram índice significativo de toxidez $\left(\mathrm{DL}_{50} \cong 1500\right)$.

A fração 11-12 foi cristalizada em metanol e obteve-se um material branco cristalino $(55 \mathrm{mg})$ constituído de uma mistura dos esteróides $\beta$-sitosterol e estigmasterol e do éster metílico 1.

A fração 26-32 (21,7g) foi submetida a fracionamento cromatográfico em coluna de sílicagel, usando-se clorofórmio e metanol como eluentes. Foram coletadas 64 frações. A fração 7 (278,0 mg), que revelou o maior grau de pureza, foi submetida à purificação através de cromatografia em camada delgada preparativa e obteve-se o alcalóide 2 (50 mg, PF $230-231{ }^{\circ} \mathrm{C}$ ).

A fração 36-43 foi dissolvida em metanol a quente e após o resfriamento da solução forneceu o alcalóide $\mathbf{3}$ como um precipitado amarelo $(4,0 \mathrm{~g})$, pf $178-180{ }^{\circ} \mathrm{C},[\alpha]_{\mathrm{D}}:-13,95$ (c 0,043, MeOH).

O extrato SGCH $(560 \mathrm{mg})$ foi fracionado através de cromatografia em camada preparativa circular (Chromatotron), usando-se clorofórmio puro como eluente. Obteve-se 25 frações, que foram reunidas em 9 grupos após análise através de cromatografia em camada fina com diferentes eluentes. A análise das frações com espectros IV e RMN ${ }^{1} \mathrm{H}$ permitiu identificar uma mistura de material alifático e uma mistura de sitosterona e estigmasterona.

Metilação de 3: O alcalóide 3 (100 mg) foi dissolvido em metanol e adicionou-se diazometano em excesso. Após a evaporação do solvente e a análise dos dados fornecidos por espectros de $\mathrm{RMN}{ }^{1} \mathrm{H}$ $\mathrm{e}^{13} \mathrm{C}$ do produto confirmou-se a formação do lialosídeo $4(100 \mathrm{mg})^{16}$, em acordo com a previsão baseada em citação da literatura ${ }^{8}$.

Acetilação de 3: $\mathrm{O}$ alcalóide $\mathbf{3}(100 \mathrm{mg}$ ) foi dissolvido em piridina (2,0 $\mathrm{mL})$ e adicionu-se anidrido acético $(2,0 \mathrm{~mL})$ e, posteriormente, 2 mg de pirrolidinopiridina (PP). A solução foi submetida a refluxo durante $3 \mathrm{~h}$. Após este período e o resfriamento da solução, adicionou-se água gelada $(4,0 \mathrm{~mL})$ e extraiu-se com clorofórmio $(3 \times 10,0 \mathrm{~mL})$. A solução orgânica foi lavada com $\mathrm{HCl}(10 \%)$ e depois com água. Após secagem com sulfato de sódio anidro, o solvente foi evaporado e o resí- 
Tabela 3. Dados de $\mathrm{RMN}{ }^{1} \mathrm{H}(400 \mathrm{MHz})$ e ${ }^{13} \mathrm{C}(100 \mathrm{MHz})$ do produto acetilado $\mathbf{5}^{*}$

\begin{tabular}{|c|c|c|c|c|}
\hline \multirow[b]{2}{*}{$\mathbf{C}$} & \multicolumn{2}{|c|}{ HMQC } & \multirow{2}{*}{$\begin{array}{c}\text { HMBC } \\
{ }^{2} \mathrm{~J}_{\mathrm{CH}} \mathrm{e}^{3} \mathrm{~J}_{\mathrm{CH}}\end{array}$} & \multirow[b]{2}{*}{${ }^{1} \mathrm{Hx}{ }^{1} \mathrm{H}-\mathrm{COSY}$} \\
\hline & $\delta_{\mathrm{C}}$ & $\delta_{\mathrm{H}}[$ mult., $\mathrm{J}(\mathrm{Hz})]$ & & \\
\hline 2 & 137,3 & - & & \\
\hline 3 & 143,7 & - & $\mathrm{H}^{*} ; \mathrm{H}-5$ & \\
\hline 5 & 141,9 & $8,40(\mathrm{~d} ; 5,2)$ & & H-6 \\
\hline 6 & 113,8 & $7,80(\mathrm{~d} ; 5,2)$ & $\mathrm{H}-5$ & $\mathrm{H}-5$ \\
\hline 7 & 132,8 & - & H-5 & \\
\hline 8 & 123,2 & - & $\mathrm{H}-10$ & \\
\hline 9 & 120,6 & $8,20(\mathrm{~d} ; 8,0)$ & H-11 & H-10 \\
\hline 10 & 123,8 & $7,40(\mathrm{t} ; 8,0)$ & H-12 & H-9; H-11 \\
\hline 11 & 130,1 & $7,60(\mathrm{t} ; 8,0)$ & $\mathrm{H}-9$ & H-10; H-12 \\
\hline 12 & 118,3 & $8,60(\mathrm{~d} ; 8,0)$ & H-10 & H-11 \\
\hline 13 & 140,6 & - & $\mathrm{H}-11 ; \mathrm{H}-9$ & \\
\hline 14 & 31,1 & $2,20(\mathrm{~s})$ & & \\
\hline 15 & 32,7 & $3,50(\mathrm{~m})$ & $\mathrm{H}-17$ & $\mathrm{H}-20$ \\
\hline 16 & 112,6 & - & H-17 & \\
\hline 17 & 150,9 & $7,80(\mathrm{~d} ; 2,8)$ & $\mathrm{H}-21$ & H-15 \\
\hline 18 & 132,2 & & H-17 & H-19; H-20 \\
\hline \multirow[t]{2}{*}{19} & 121,2 & $5,40(\mathrm{dm} ; 16,8)$ & & H-18 H-18 \\
\hline & & $5,30(\mathrm{dm} ; 13,2)$ & & \\
\hline 20 & 45,3 & $2,80(\mathrm{~m})$ & $2 \mathrm{H}-19$ & H-15; H-18; H-21 \\
\hline 21 & 96,0 & $5,30(\mathrm{~d} ; 6,4)$ & $\mathrm{H}-17$ & $\mathrm{H}-20$ \\
\hline 22 & 166,3 & & $\mathrm{H}-17$ & \\
\hline 1 ' & 95,6 & $4,90(\mathrm{~d} ; 8,0)$ & $\mathrm{H}-2$ & H-2' \\
\hline 2 & 70,4 & $4,80(\mathrm{t} ; 8,0)$ & H-3, & H-1'; H-3' \\
\hline 3 , & 72,0 & $5,10(\mathrm{t} ; 9,6)$ & $\mathrm{H}-2$ & H-2'; H-4', \\
\hline 4 & 67,8 & $5,00(\mathrm{t} ; 9,6)$ & $\mathrm{H}-3$ & H-3'; H-5' \\
\hline 5 & 72,1 & $3,70(\mathrm{dm} ; 10,0)$ & H-4'; & H-6'; H-4' \\
\hline \multirow[t]{2}{*}{6} & 61,5 & $4,10(\mathrm{dm} ; 12,0), \mathrm{H}-6 ’ \mathrm{~b}$ & & H-5'; H-6'a \\
\hline & & $4,30(\mathrm{dd} ; 12,0 ; 6,0)$, H-6'a & & H-5'; H-6’b \\
\hline $\mathrm{H}_{3} \mathrm{CCO}$ & 170,$4 ; 169,8 ; 169,2 ; 168,7$ & - & $\mathrm{H}_{3} \mathrm{C}-\mathrm{CO} ; \mathrm{H}-3^{\prime} ; \mathrm{H}-4{ }^{\prime}$ & \\
\hline $\mathrm{H}_{3}^{3} \mathrm{CCO}$ & 20,$7 ; 20,5 ; 20,4 ; 19,9$ & 2,$10 ; 2,05 ; 2,00 ; 1,80$ & & \\
\hline
\end{tabular}

*Os sinais de átomos de carbono $\mathrm{C}, \mathrm{CH}$ e $\mathrm{CH}_{3}$ foram reconhecidos pela análise comparativa dos espectros de $\mathrm{RMN}{ }^{13} \mathrm{C}-\mathrm{HBBD}$ e $\mathrm{RMN}{ }^{13} \mathrm{C}$ DEPT. Os deslocamentos químicos e constantes de acoplamento ( $\mathrm{J}$ em Hz entre parenteses) dos sinais de hidrogênio foram deduzidos do espectro 1D de RMN ${ }^{1} \mathrm{H}$.

duo foi filtrado em coluna de sílicagel, eluída com $\mathrm{CHCl}_{3}+\mathrm{MeOH}$ (9:1). A fração 5 (71,6 mg) foi fracionada em camada delgada preparativa de sílica gel (eluente acetato de etila). As frações 3 (10,3 mg) e 4 (19,5 $\mathrm{mg}$ ) deste fracionamento revelaram-se puras através de análise por TLC em diferentes eluentes. Estes produtos apresentaram-se pastosos e foram caracterizados como sendo os derivados acetilados $\mathbf{4 a}$ e $\mathbf{5}$.

\section{AGRADECIMENTOS}

Os autores agradecem ao CNPq, FAPERJ e CAPES pelas bolsas e apoio financeiro concedidos, aos Prof. P.G. Filho e S. J. da Silva Neto, Departamento de Botânica, Instituto de Biologia, Universidade Federal Rural do Rio de Janeiro (UFRRJ), Seropédica, Rio de Janeiro, Brasil, pela coleta e identificação botânica do espécimen usado para investigação.

\section{REFERÊNCIAS}

1. Lewis, W.H.; Elvin-Lewis, M. P. F. Em Plants and Dental Care Among the Jivaro of the Upper Amazon Basin. Advances in Economic Botany Ethnobotany in the Neotropics; France, G. T.; Kallunkie, J. A., eds.; Botanical Garden Bronx: NY, 1984, 1, 53.
2. Castro, O.; Lopes, V. J.; Ing. Cienc. Quim. 1986, 10, 56.

3. Hasbun, C. P.; Calseron, M.; Castro, O; Gacs-Baitz, E.; Delle Monache, G.; Delle Monache, F.; Tetrahedron Lett. 1989, 30, 6199.

4. Kelly, T.; Xu, W.; Sundaresan, J.; Tetrahedron Lett. 1993, 34, 6173.

5. Castaneda, P.; Albor, C.; Matar, R.; Bye, R.; Linares, E.; Fitoterapia 1991, 62,366 .

6. Arnason, T.; Morand, P.; Salvador, J.; Reyes, I.; Lanbert, J.; Towers, G.H.N.; Phytochemistry 1983, 22, 594.

7. Buckingham, J., ed.; Dictionary of Natural Products, Chapman \& Hall: London, 1994, p. 1515.

8. Aimi, N.; Tsuyuki, T.; Murakami, H.; Sakai, S.; Haginiwa, J.; Tetrahedron Lett. 1985, 26, 5299.

9. Goulart, M. O. F.; Sant'Ana, A. E. G.; Lima, R. A. de; Cavalcante, S. H.; Carvalho, M. G. de; Braz-Filho, R.; Quim. Nova 1993, 16, 95.

10. Wright, J. L. C.; McInnes, A. G.; Shimizu, S.; Smith, D. G.; Walter, J. A.; Idler, D.; Khalil, W.; Can. J. Chem. 1979, 56, 1898.

11. Thimmerman, B. N.; Hoffman, J. J.; Jola, S. D.; Sehram, R. H.; Klencn, R.E.; Bates, R. B;. J. Nat. Prod. 1983, 46, 356.

12. Migliuolo, A.; Piccialli, V.; Sica, D.; J. Nat. Prod. 1990, 53, 1262.

13. Joshi, K. C.; Indian J. Chem. 1995, 12, 903.

14. Levesque, J.; Jacquesy, R.; Foucher, J. P.; Tetrahedron 1982, 38, 1417.

15. Boonyaratavej, S.; Tantayanontha, S.; Kitchanachai, P.; J. Nat. Prod. 1992, $55,1761$.

16. Valverde, J.; Tamayo, G.; Hesse, J.; Phytochemistry 1999, 52, 1485.

17. Siqueira, J. M. de; Bomm, M. D.; Pereira, N. F. G.; Garcez, W. S.; Boaventura, M. A. D.; Quim. Nova 1998, 21, 557. 